\title{
The effects of three novel probiotics isolated from the Korean fermented food Kimchi on the stress-induced defecation of rats
}

\author{
Min Ji Kim ${ }^{1}$, Yang Won Min ${ }^{1}$, Chansu Lee ${ }^{1}$, Yeon Sil Jang ${ }^{1}$, Cheol Hyun Kim², \\ Dong Kyung Chang ${ }^{1}$
}

${ }^{1}$ Department of Internal Medicine, Samsung Medical Center, Sungkyunkwan University School of Medicine, Seoul, Korea

${ }^{2}$ Department of Animal Resource \& Science, Dankook University, Cheonan, Korea

Received: October 2, 2019

Revised: February 12, 2020

Accepted: February 17, 2020

Corresponding author:

Yang Won Min

Department of Internal

Medicine, Samsung Medical

University School of Medicine,

81 Irwon-ro, Gangnam-gu,

Seoul 06351, Korea

Tel: +82-2-3410-3409

E-mail: yangwonee@gmail.com
Center, Sungkyunkwan

\begin{abstract}
Purpose: The interest in novel probiotics that improve symptoms of irritable bowel syndrome (IBS) has been growing trend. This study aims to investigate the probiotic effect of three kinds of Lactobacillus strain that are isolated from kimchi.

Methods: We used Wister strain rats, male sex. The rat subjects were subdivided into four groups by which to be administered Lactobacillus casei DK128, Lactobacillus paracasei DK121, Lactobacillus platarum DK119 or vesicle only (control). Each Lactobacillus $\left(1 \times 10^{9}\right.$ colony forming unit) was administered by oral gavage once daily for 10 days. At day 10 , the rats were stressed by being placed in confinement in a restraining stress cage for 1 hour. The stool of the animals were collected after the stress test. The stool weight was recorded and measured in grams, then, each data were transformed to a percentage as compared with the mean stool weight of same age in control group. The stool consistency was scored 0 to 4 ( 0 , normal; 2 , loose stool; 4 , diarrhea). We compared the stool weight and consistency between the treatments.

Results: The total stool weight of the rats was significantly reduced in the $L$. casei DK128 group (65\%, $\mathrm{P}=0.0368)$ and the $L$. paracasei $\mathrm{DK} 121$ group $(64 \%, \mathrm{P}=0.0138)$, as compared to the control group (100\%). The stool consistency of the L. paracasei DK121 group was noted as being better than the control group ( 2 vs. $4, P=0.0272$ ).

Conclusion: Our findings suggest that L. casei DK128 and L. paracasei DK121 has the characteristics of probiotics that could control symptoms of IBS.
\end{abstract}

Keywords: Irritable bowel syndrome; Kimchi; Probiotics; Rats; Stress

\section{INTRODUCTION}

distributed under the terms of the Creative Commons Attribution Non-Commercial License (https:// creativecommons.org/licenses/ by-nc/4.0/).
Irritable bowel syndrome (IBS) is a common gastrointestinal disorder which is characterized by recurrent abdominal pain associated with alteration in bowel habits [1-3]. It has been found that there are differences in the gut luminal microbiota between patients with IBS and a 


\section{PRECISION AND FUTURE MIEDICINE}

Effects of probiotics from Kimchi

healthy control, also, the pathogenesis of IBS may be related to an alteration in the individual's gut microbiota $[4,5]$. Accordingly, therapeutic options that have potential to alter intestinal bacterial composition and their metabolic products have been tested to help alleviate the symptoms of IBS, including the use of probiotics which are available in some food products.

It is emphasized that there are numerous reports that probiotics may beneficial in the managing the symptoms of IBS [6-8]. However, the efficacy of probiotics has been proven individually and the therapeutic benefits of strain of probiotics cannot extended to other strains [9]. Therefore, there are requests for novel probiotics to be revealed and identified for the benefit of treatment for humans with IBS. Recently, there have been numerous studies for mining the effective species and strains that have been conducted, and the results are promising.

Kimchi, which is a traditional Korean spicy based fermented food, is considered to be a good source of potentially beneficial and useful lactic acid bacteria (LAB) [10]. A previous study was undertaken to isolate and identify the strains of potentially probiotic LAB as taken from samples of kimchi, whereby three strains were found to be the promising strains, namely Lactobacillus platarum DK119, Lactobacillus paracasei DK121, and Lactobacillus casei DK 128. This referenced showed relatively good results in tolerance to acid and bile salt, cold-shock induced cryo-tolerance, and beta-galactosidase and proteolytic activity [11].

The aim of the present study is to investigate the probiotic potency of these three kinds of Lactobacillus strain, through access the effects on stress-induced abnormal defecation of rats.

\section{METHODS}

\section{Animals}

For this study, male Wistar rats aged 11 weeks (weight 340 to $410 \mathrm{~g}$ ) and aged 8 weeks (weight 240 to $260 \mathrm{~g}$ ) were used. In the study, eight rats aged 11 weeks and 12 rats age 8 weeks were divided into four groups: each group consist of two rats aged 11 weeks and three rats aged 8 weeks. This study was reviewed and approved by the Institutional Animal Care and Use Committee of Samsung Biomedical Research Institute (IACUC No.20170216002). The Samsung Biomedical Research Institute is an Association for Assessment and Accreditation of Laboratory Animal Care International accredited facility and the study abided by the Institute of Laboratory Animal Resources guidelines.

\section{Lactobacillus strains}

Three Lactobacillus strains, L. platarum DK119, L. paracasei DK121, and L. casei DK128, which are isolated from Korean fermented food Kimchi were used in this study. These strains were isolated, selected and identified through previous study conducted by Cho et al. [11]. The samples from homemade and commercial kimchi were mixed with phosphate-buffered saline (PBS) and diluted with $0.85 \%$ saline, then each dilution was spread onto MRS agar (De Man, Rogosa and Sharpe agar) plate containing $0.02 \%$ sodium azide. The plates were incubated for 48 hours and representative colonies were picked from plates. There are several studies noting that probiotics including the Lactobacillus strain has effective symptom relief in IBS patients [12-14]. Gram positive, non-motile and rod shaped bacteria showing phenotypic characters similar to Lactobacillus species on MRS agar media being able to growth at $\mathrm{pH} 4.4$ were selected for further test.

Selected strains were tested bile salt tolerance, acid tolerance, and cryo-tolerance, for requirement of the factors that could affect the viability of probiotic bacteria, including gastric acid, bile salt and refrigerated storage. Also, beta-galactosidase and proteolytic activity were tested for commercial use as probiotics and starter culture in dairy products. Three selected isolates that showed relatively good results, were desingnated as DK119, DK121, and DK128. Identification was confirmed by using an API kit and 16s rRNA gene sequencing. DK119 was identified as L. plantarum, DK121 as L. paracasei, and DK128 as L. casei.

\section{Protocols}

In this experiment, the rats were divided into four groups; three treatment groups that each treated with $L$. platarum DK 119, L. paracasei DK121, and L. casei DK 128, and the control group. In this study, rats in the treatment groups were administered a suspension of Lactobacillus $\left(1 \times 10^{9}\right.$ colony forming unit) using oral gavage at the same time once daily for 10 days, whereas the control group received a suspension of PBS for the same period (Fig. 1). At day 10, the rats were

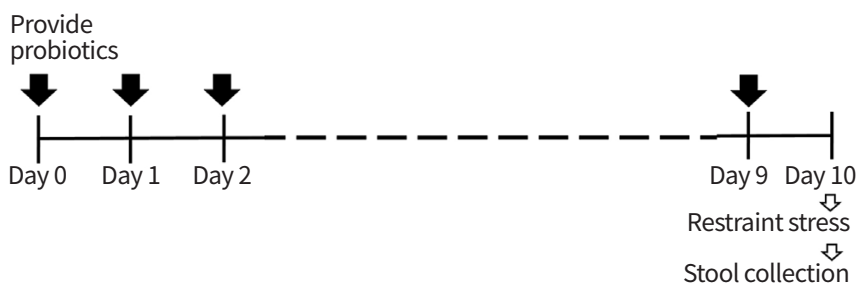

Fig. 1. Study protocol. 
stressed by being placed in a controlled area of confinement in a restraining stress cage (Fig. 2) for 1 hour. The stools were collected 1 hour after the initiation of restraint stress.

\section{Measurement}

The outcomes and measurements for this experiment are noted as the results of the stool weight and consistency. The stool weight was measured using the total stool collected during 1 hour after a stress was induced on the rats. The stool consistency result was scored from 0 to 4 ( 0 , normal; 2 , loose stool; 4, watery). To check the growth of rats, the body weight of each of the rats was measured every other day and noted during the experiment.

\section{Statistical analysis}

We compared the stool weight and consistency of data be-

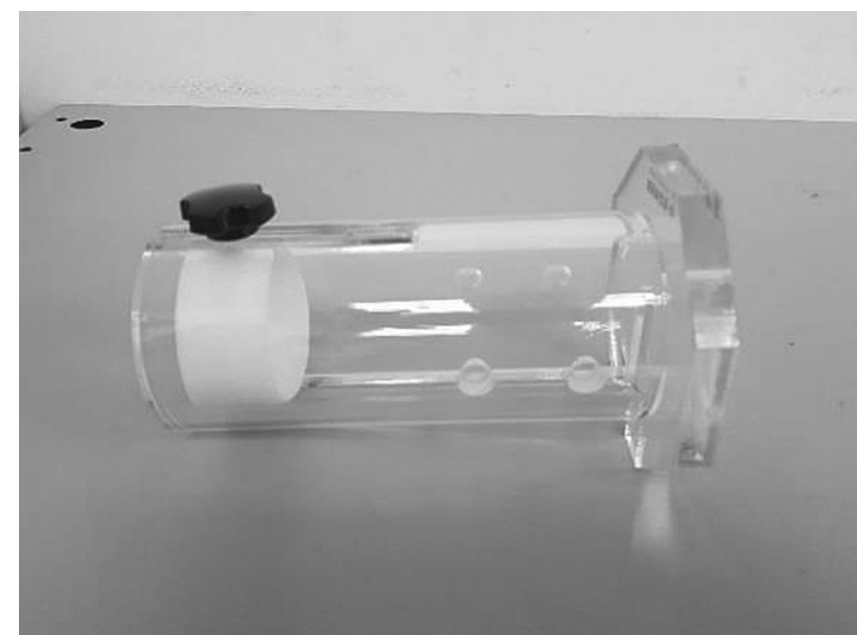

Fig. 2. Restraint stress cage.

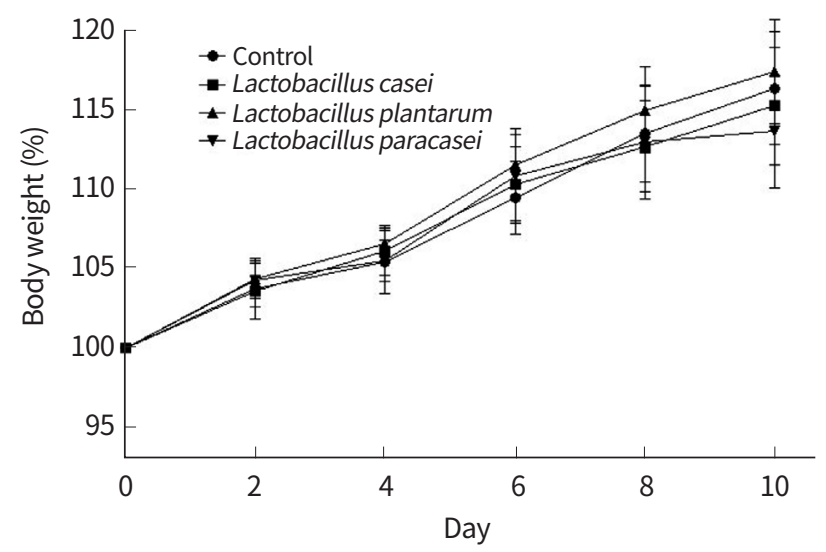

Fig. 3. The body weight of rats. Because the initial body weight of rats was different in each group, it was analyzed by percentile increased weight. There were no significant differences between the groups. tween the treatment and the experiment control group, to investigate the effects of probiotics on the stress-induced defecation in rats. Because the initial body weight of rats were noted as different in each group, the data was analyzed by a percentile increased weight. In these terms, the mean of stool weights of each age in control group were set up as $100 \%$, then, each stool weight data were transformed to a percentage compared with the mean of same age. The stool weight data were presented as percentage and the median with interquartile range (IQR). The stool consistencies are presented as median with IQR. In other words, the outcomes were compared between the treatment group and the control by the use of a Mann-Whitney U-test model, using the statistical software GraphPad Prism version 5 (GraphPad Software, La Jolla, CA, USA). The statistical significance was set at a level of $\mathrm{P}<0.05$.

\section{RESULTS}

\section{Body weight of rats}

It is noted that the body weight of the study rats were linearly increased during experiment. Additionally, it is noted that the weight gain did not differ between the four groups (Fig. 3). No rats were noted to have died during the experiment.

\section{The total stool weight}

In this study, the average stool weights in control group were $3.6 \mathrm{~g}$ in age 11 weeks, and $2 \mathrm{~g}$ in age 8 weeks. The median percentages of stool weight were $65.1 \%$ (IQR, $51.8 \%$ to $82.5 \%$ ) in

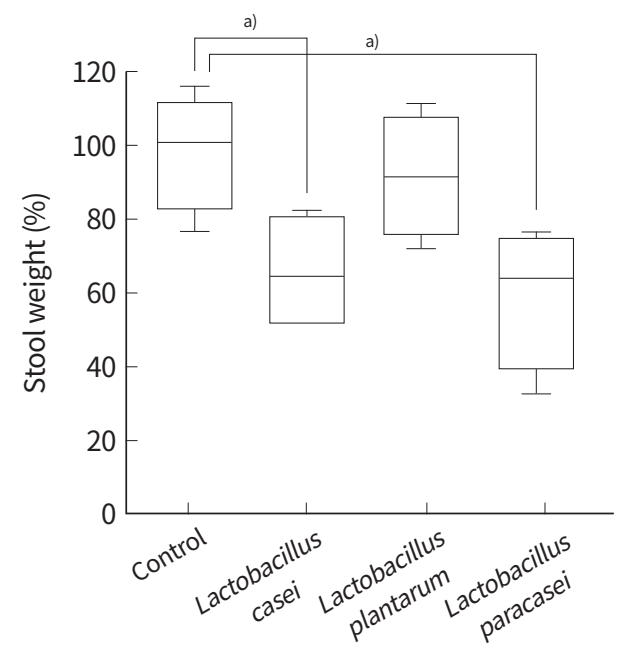

Fig. 4. The total stool weight between groups. Each data was transformed to a percentage of mean of the same aged rat's total stool weight in the control group. Data were presented in median with interquartile range. a) $\mathrm{P}<0.05$ by Mann-Whitney U-test. 


\section{PRECISION AND FUTURE MIEDICINE}

Effects of probiotics from Kimchi

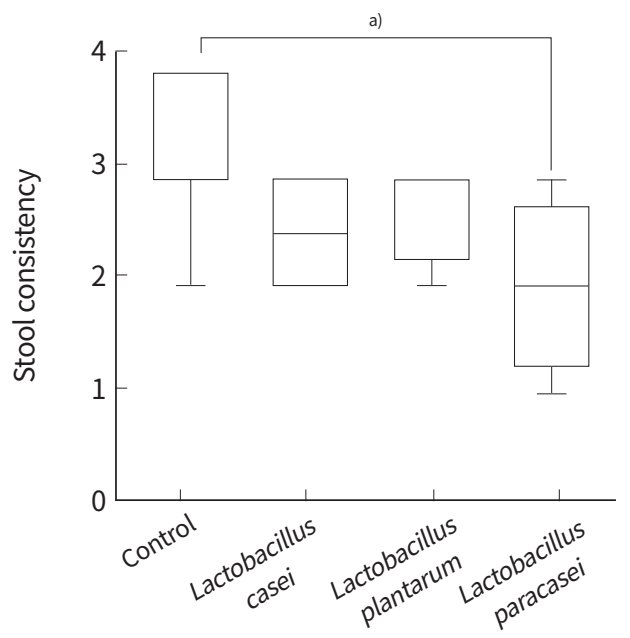

Fig. 5. The stool consistency between group. Data were presented in median with interquartile range. ${ }^{\text {a) }} \mathrm{P}<0.05$ by Mann-Whitney U-test.

L. casei DK128, 93.7\% (IQR, 76.1\% to $110.7 \%$ ) in L. platarum DK121, and 64.3\% (IQR, 38.0 to $75.6 \%$ ) in L. paracasei DK121. The total stool weight was significantly reduced in $L$. casei DK128 group $(P=0.0368)$ and $L$. paracasei $D K 121$ group $(P=$ 0.0138 ) compared to the control group (Fig. 4).

\section{Stool consistency}

We examined and noted that the median score of stool consistency was 3.5 (IQR, 3 to 4 ) in control group, 2.5 (IQR, 2 to 3 ) in L.casei DK128 group, 3 (IQR, 2.25 to 3) in L. plantarum DK119 group, and 2.0 (IQR, 1.25 to 2.75) in L. paracasei DK121 group. The stool consistency of $L$. paracasei group $(P=0.0272)$ was better than the control group (Fig. 5).

\section{DISCUSSION}

In this study, we aimed to prove the potent inhibitory effect of Lactobacillus stains that were isolated from kimchi on stress-induced abnormal defecation in rats. The total stool weight was significantly reduced in rats which are administered L. casei DK128 and L. paracasei DK121. Especially, where $L$. paracasei DK121 showed a better outcome at both stool weight and consistency. There were no growth interruptions nor death at the Lactobacillus administration group. The results of this study indicated that application of Lactobacillus strains that are isolated from kimchi reduced the stress-induced fecal output in rats.

We used a restrain stress model in this study to investigate probiotic potency of three Lactobacillus strains. The restrain stress model consists of forced immobilization of the animal and is applied once during testing [15]. Animal shows abnormal defection after restrain stress. This model reproduced, at least in part, the main symptoms present in IBS, visceral hypersensitivity and dysmotility $[16,17]$. The efficacy of this test is confirmed by the development of an immediate hyperalgesia [18], the inhibition of small intestinal transit, the stimulation of large intestinal activity and the incidence of an increased fecal excretion [15]. Also, it was recently demonstrated that rats underwent testing to the restrain stress presented a low-grade mucosal inflammation, with a significant increase in mast cells and eosinophilic granulocytes [19,20], that is seen to have overlapped on what is described in colonic biopsies of IBS patients [21].

This model was introduced in the scientific literature more than 30 year ago, and commonly applied to investigate potential drugs to treat IBS. For example, in the Hirata et al. [22] study, the researchers examined the pharmacological profile of ramosetron, selective 5-hydroxytryptamine 3 receptor antagonists receptor antagonist (5-HT3), for IBS-D, and ramosetron dose-dependently inhibited restraint stress induced defecation in rats. In a recent randomized controlled trial with IBS patients with diarrhea symptoms, it is noted that ramosetron has proved effective in improving stool consistency, relieving abdominal pain/discomfort, and improving the patient's health-related quality of life $[23,24]$.

Probiotics, defined as living microorganisms that have benefits for the gut tract and its immune function after being ingested [25]. There is growing evidence of the role of imbalance of the intestinal microbiota, intestinal infections, and a dysfunctional intestinal barrier in the development of IBS and its symptoms [26]. Therefore, the therapeutic potential of probiotics has gathered a lot of interest. Gut microbiota known to be influenced the bidirectional communication between the enteric nervous system and central nervous system, modulated the physiological gut functions [27]. Probiotics altered composition and/or activity of gut microbiota and may alter the physiological gut functions, including intestinal motility and hypersensitivity [28]. Numerous clinical trials have investigated the effects of probiotics in patients with IBS $[29,30]$. However, when interpreting trials of probiotics in any research area, the results found with one probiotic species cannot be extrapolated to another result or test. Different strains may have very different immunological and enzymatic effects within the host [4]. Therefore, studies such as the present experiment for mining the novel probiotics and their characteristics are needed.

In this study, the results suggests the potential utility of 
Lactobacillus strain in conditions of stress-associated increased defecation and altered bowel function, and it suggested the probiotic potency of the strains. Potent probiotic effect of Lactobacillus strain isolated from kimchi that investigated in this study might help relieve symptoms of IBS patients.

There are several limitations in our study. First, the sample size is small for this study. The results might be distorting of the analysis, and results in a different study with a larger sample size may provide different conclusions, such as in the case of different animal handling or gavaging. However, the results in this experiment showed significant differences despite the incidence of a small sample size, and it implies that the evidence of a potent inhibitory effect of Lactobacillus strain is suggestive. Second, there are issues that these findings are applicable to human. Further studies are required to ascertain the potent effect of Lactobacillus strain as use for a probiotics therapy in human and IBS patients. The restrain stress model misses the characterizing clinical condition of IBS such as with a chronic course, and there are interspecies differences. However, this experiment is an animal study for investigating the safety and beneficial effect of potent candidate of novel probiotics, where it is suggested that further clinical trials will be studied and reviewed in the future.

In conclusion, our findings indicate that L. casei DK128 and L. paracasei DK121 have probiotic potency as relieving symptoms or IBS.

\section{CONFLICTS OF INTEREST}

No potential conflict of interest relevant to this article was reported.

\section{ACKNOWLEDGMENTS}

This work was supported by Korea Institute of Planning and Evaluation for Technology in Food, Agriculture, Forestry and Fisheries (IPET) through High Value-Added Food Technology Development Program, funded by Ministry of Agriculture, Food and Rural Affairs (MAFRA) (316061-3).

\section{ORCID}

Min Ji Kim https://orcid.org/0000-0002-1059-5402

Yang Won Min https://orcid.org/0000-0001-7471-1305

Chansu Lee https://orcid.org/0000-0003-3992-9645

Yeon Sil Jang
Cheol Hyun Kim https://orcid.org/0000-0002-1905-9948

Dong Kyung Chang https://orcid.org/0000-0001-8925-4629

\section{AUTHOR CONTRIBUTIONS}

Conception or design: YMW, CL.

Acquisition, analysis, or interpretation of data: MJK, YWM, CL, YSJ, CHK.

Drafting the work or revising: MJK.

Final approval of the manuscript: MJK, YWM, CL, YSJ, CHK, DKC.

\section{REFERENCES}

1. Mearin F, Lacy BE, Chang L, Chey WD, Lembo AJ, Simren M, et al. Bowel disorders. Gastroenterology 2016;150: 1393-407.e5.

2. Chey WD, Kurlander J, Eswaran S. Irritable bowel syndrome: a clinical review. JAMA 2015;313:949-58.

3. Amouretti M, Le Pen C, Gaudin AF, Bommelaer G, Frexinos J, Ruszniewski P, et al. Impact of irritable bowel syndrome (IBS) on health-related quality of life (HRQOL). Gastroenterol Clin Biol 2006;30:241-6.

4. Parkes GC, Brostoff J, Whelan K, Sanderson JD. Gastrointestinal microbiota in irritable bowel syndrome: their role in its pathogenesis and treatment. Am J Gastroenterol 2008; 103:1557-67.

5. Distrutti E, Monaldi L, Ricci P, Fiorucci S. Gut microbiota role in irritable bowel syndrome: new therapeutic strategies. World J Gastroenterol 2016;22:2219-41.

6. Cui S, Hu Y. Multistrain probiotic preparation significantly reduces symptoms of irritable bowel syndrome in a double-blind placebo-controlled study. Int J Clin Exp Med 2012;5:238-44.

7. Whorwell PJ, Altringer L, Morel J, Bond Y, Charbonneau D, O'Mahony L, et al. Efficacy of an encapsulated probiotic Bifidobacterium infantis 35624 in women with irritable bowel syndrome. Am J Gastroenterol 2006;101:1581-90.

8. Yuan F, Ni H, Asche CV, Kim M, Walayat S, Ren J. Efficacy of Bifidobacterium infantis 35624 in patients with irritable bowel syndrome: a meta-analysis. Curr Med Res Opin 2017;33:1191-7.

9. Dai C, Zheng CQ, Jiang M, Ma XY, Jiang LJ. Probiotics and irritable bowel syndrome. World J Gastroenterol 2013; 19:5973-80.

10. Park KY, Jeong JK, Lee YE, Daily JW 3rd. Health benefits of kimchi (Korean fermented vegetables) as a probiotic food. 


\section{PRECISION AND FUTURE MIEDICINE}

Effects of probiotics from Kimchi

J Med Food 2014;17:6-20.

11. Cho YH, Hong SM, Kim CH. Isolation and characterization of lactic acid bacteria from kimchi, Korean traditional fermented food to apply into fermented dairy products. Korean J Food Sci Anim Resour 2013;33:75-82.

12. O'Mahony L, McCarthy J, Kelly P, Hurley G, Luo F, Chen K, et al. Lactobacillus and bifidobacterium in irritable bowel syndrome: symptom responses and relationship to cytokine profiles. Gastroenterology 2005;128:541-51.

13. Ducrotte P, Sawant P, Jayanthi V. Clinical trial: Lactobacillus plantarum 299v (DSM 9843) improves symptoms of irritable bowel syndrome. World J Gastroenterol 2012; 18:4012-8.

14. Niedzielin K, Kordecki H, Birkenfeld B. A controlled, double-blind, randomized study on the efficacy of Lactobacillus plantarum $299 \mathrm{~V}$ in patients with irritable bowel syndrome. Eur J Gastroenterol Hepatol 2001;13:1143-7.

15. Williams CL, Villar RG, Peterson JM, Burks TF. Stress-induced changes in intestinal transit in the rat: a model for irritable bowel syndrome. Gastroenterology 1988;94:61121.

16. Wang Y, Bi Z, Wang E, Sun B, Zheng Y, Zhong LL, et al. Rodent model of irritable bowel syndrome. Int J Gastroenterol Disord Ther 2017;4:131.

17. Vannucchi MG, Evangelista S. Experimental models of irritable bowel syndrome and the role of the enteric neurotransmission. J Clin Med 2018;7:E4.

18. Gue M, Del Rio-Lacheze C, Eutamene H, Theodorou V, Fioramonti J, Bueno L. Stress-induced visceral hypersensitivity to rectal distension in rats: role of CRF and mast cells. Neurogastroenterol Motil 1997;9:271-9.

19. Traini C, Evangelista S, Girod V, Faussone-Pellegrini MS, Vannucchi MG. Changes of excitatory and inhibitory neurotransmitters in the colon of rats underwent to the wrap partial restraint stress. Neurogastroenterol Motil 2016; 28:1172-85.

20. Traini C, Evangelista S, Girod V, Faussone-Pellegrini MS, Vannucchi MG. Repeated otilonium bromide administration prevents neurotransmitter changes in colon of rats underwent to wrap restraint stress. J Cell Mol Med 2017; 21:735-45.

21. Bashashati M, Moossavi S, Cremon C, Barbaro MR, Moraveji S, Talmon G, et al. Colonic immune cells in irritable bowel syndrome: a systematic review and meta-analysis. Neurogastroenterol Motil 2018;30:e13192.

22. Hirata T, Keto Y, Funatsu T, Akuzawa S, Sasamata M. Evaluation of the pharmacological profile of ramosetron, a novel therapeutic agent for irritable bowel syndrome. J Pharmacol Sci 2007;104:263-73.

23. Fukudo S, Ida M, Akiho H, Nakashima Y, Matsueda K. Effect of ramosetron on stool consistency in male patients with irritable bowel syndrome with diarrhea. Clin Gastroenterol Hepatol 2014;12:953-9.

24. Lee KJ, Kim NY, Kwon JK, Huh KC, Lee OY, Lee JS, et al. Efficacy of ramosetron in the treatment of male patients with irritable bowel syndrome with diarrhea: a multicenter, randomized clinical trial, compared with mebeverine. Neurogastroenterol Motil 2011;23:1098-104.

25. Food and Agriculture Organization of the United Nations and World Health Organization Expert Consultation Report. Evaluation of health and nutritional properties of probiotics in food including powder milk with the live lactic acid bacteria [Internet]. Rome (IT): Food and Agriculture Organization of the United Nations; c2020 [cited 2020 Feb 25]. Available from: http://www.fao.org/3/ a-a0512e.pdf.

26. Clarke G, Cryan JF, Dinan TG, Quigley EM. Review article: probiotics for the treatment of irritable bowel syndrome: focus on lactic acid bacteria. Aliment Pharmacol Ther 2012;35:403-13.

27. Holtmann GJ, Ford AC, Talley NJ. Pathophysiology of irritable bowel syndrome. Lancet Gastroenterol Hepatol 2016;1:133-46.

28. Kanazawa M, Hongo M, Fukudo S. Visceral hypersensitivity in irritable bowel syndrome. J Gastroenterol Hepatol 2011;26 Suppl 3:119-21.

29. Kajander K, Hatakka K, Poussa T, Farkkila M, Korpela R. A probiotic mixture alleviates symptoms in irritable bowel syndrome patients: a controlled 6-month intervention. Aliment Pharmacol Ther 2005;22:387-94.

30. Williams EA, Stimpson J, Wang D, Plummer S, Garaiova I, Barker ME, et al. Clinical trial: a multistrain probiotic preparation significantly reduces symptoms of irritable bowel syndrome in a double-blind placebo-controlled study. Aliment Pharmacol Ther 2009;29:97-103. 